\title{
Numerical simulation of the sea bottom modifications behind a T-head groin
}

\author{
MARCO TAMBURRINO, FRANCESCO GALLERANO \\ Department of Civil, Constructional and Environmental Engineering \\ "Sapienza" University of Rome \\ Via Eudossiana 18, 00184 \\ ITALY
}

\begin{abstract}
In this paper, we simulate the sea bottom modifications produced by the presence of a T-head groin. We present a simulation model of sea bottom modifications composed of two sub-models: a two-dimensional phase-resolving model that simulate the variation of the fluid dynamic variables inside the wave; a second submodel to simulate the sea bottom modifications, in which the suspended sediment concentration is calculated by the wave-averaged advection-diffusion equation. The fluid motion equation and the concentration equation are expressed in a new contravariant formulation. The velocity fields from deep water up to just seaward of the surfzone are simulated by a new integral contravariant form of the Fully Nonlinear Boussinesq Equations. The new integral form of the proposed continuity equation does not contain the dispersive term. The Nonlinear Shallow Water Equations, expressed in an integral contravariant form, are solved in order to simulate the breaking wave propagation. The momentum equation, integrated over the turbulent boundary layer, is solved to calculate the near-bed instantaneous flow velocity and the intra-wave hydrodynamic quantities. Starting from the contravariant formulation of the advection-diffusion equation for the suspended sediment concentration, it is possible to calculate the sea bottom modification. The advective sediment transport terms in the advection-diffusion equation are formulated according to a quasi-three-dimensional approach.
\end{abstract}

Key-Words: - Phase-resolving model, undertow, intra-wave quantities, concentration equation, sediment transport, sea bottom modification.

Received: September 2, 2019. Revised: February 27, 2020. Accepted: March 17, 2020. Published: March 31, 2020.

\section{Introduction}

The sea bottom modifications produced by a coastal defence structure, in the literature, is carried out by the simulation of the velocity fields and of the suspended sediment concentration. The threedimensional simulation of the velocity fields [1-3] requires considerable computational time for the long-term sea bottom simulations. Consequently, the motion equations and the concentration equation are depth-averaged.

The two-dimensional phase-resolving models (that are not averaged over the wave period) use the Boussinesq equations, obtained by defining the depth dependence of the variables, and by depth integrating the motion equations.

Shock-capturing schemes for numerical integration of the Fully Nonlinear Boussinesq Equations (hereinafter called FNBE's) [4,5] and nonlinear shallow-water equation (hereinafter called NSWE) models allow explicit simulations of wave breaking [6].

Sea bottom modifications in the coastal region are produced by complex hydrodynamic processes; among these, the undertow plays a fundamental role in the transport of solid particles in the offshore direction. The undertow consists of a circulation in the vertical plane in which the near-bed current velocities are off-shore directed in the surf zone. In order to simulate the undertow Lynett [7] proposed a correction to the vertical distribution of the horizontal velocity calculated by depth-averaged motion equations.

The hydrodynamic quantities that vary in the wave period drive the resuspension of the solid particles and their transport and settling. The wave period variability of the fluid dynamic quantities, the wave-current interaction can be taken into account by FNBE's/NSWE models.

The particular form of the FNBE's present in the literature, due to the presence of the dispersive terms in the continuity equation, and the schemes by which these terms are discretized, produce a lack of a high level of accuracy in the wave motion simulation.

In the works present in the literature, the improvement of the dispersive properties of the standard forms of the FNBE's is due to the adoption of the velocity at an arbitrary distance from the still water level, as dependent variables, instead of the depth-averaged velocity. This choice entailed the 
appearance of the dispersive term in the continuity equation.

In the works present in the literature [8-11], the dispersive term, that appears in the continuity equation, is discretized with a second-order cellcentered finite difference scheme consistently with the finite difference scheme of the hybrid scheme (finite volume - finite difference). The discretization of this dispersive term by a second-order cellcentered finite difference scheme, introduces truncation errors into the solution that can reduce the local accuracy of the numerical scheme and induce oscillations in the numerical solution.

We present a simulation model of sea bottom modifications composed of two sub-models: a twodimensional phase-resolving model that simulate the variation of the fluid dynamic variables inside the wave period and that takes into account the undertow; a second sub-model to simulate the sea bottom modifications, in which the suspended sediment concentration is calculated by the wave-averaged advection-diffusion equation. The fluid motion equation and the concentration equation are expressed in a new contravariant formulation.

In this paper, a new integral form of the FNBE's is proposed in order to simulate hydrodynamic fields from deep water up to just seaward of the surf zones. The abovementioned motion equations retain the term related to the second-order vertical vorticity. Breaking wave propagation in the surf zone is simulated by integrating the NSWE.

The integral form of the proposed continuity equation does not contain dispersive terms and is entirely discretized by a shock-capturing finite volume scheme: in this way, the errors due to the loworder discretization of such dispersive term are not induced into the solution.

From the horizontal velocity vertical profile, obtained by the proposed hydrodynamic model, and from the integration of the momentum equation over the wave boundary layer, the near-bed velocity, the instantaneous boundary layer thickness, the friction velocity and the bed shear stress are calculated.

The instantaneous eddy viscosity vertical distribution, under breaking waves, is calculated by taking into account the turbulence contribution due to the wave boundary layer, current and wave breaking.

Bed evolution dynamics is calculated starting from the contravariant formulation of the advectiondiffusion equation for the suspended sediment concentration. The advective sediment transport terms, that appear in the advection-diffusion equation, are formulated according to a quasi-threedimensional approach [12,13], hereinafter called Q3D; these terms are calculated starting from the depth-integrated product of the horizontal velocity and the vertical distribution of the concentration, in order to take into account the sediment transport related to the undertow. The time bottom variation is related to the contribution given by the product of the settling velocity and the difference between reference concentration and actual concentration (at a distance a from the sea bottom) and to the contribution given by the spatial variation of the bed load transport.

The paper is structured as follows. In Section 2 are proposed, the hydrodynamic and morphodynamic model. The motion equations and the concentration equation are written in integral contravariant form. In Section 3 and 4 we numerically reproduce, by the proposed model, respectively the sandbar formation and the bed evolution dynamic behind a T-head groin. In Section 5 are shown the conclusions. An appendix is found at the end of a paper.

\section{Governing equations}

\subsection{Hydrodynamic model}

We define $H=h+\eta$ as the total local water depth, where $h$ is the local still water depth and $\eta$ is free surface elevation with respect to the undisturbed free surface. By using a Taylor expansion of the velocity about an arbitrary distance from the still water surface, $\sigma$, and by assuming zero horizontal vorticity, the vertical distribution of the horizontal velocity $\vec{U}(z)$ can be written as

$$
\vec{U}(z)=\vec{u}+\vec{v}(z)
$$

where $\vec{u}$ is the horizontal velocity at an arbitrary distance from the still water level $z=\sigma$, and $\vec{v}(z)$ is defined as

$$
\begin{gathered}
\vec{v}(z)=(\sigma-z) \nabla(\nabla \cdot(h \vec{u}))+ \\
\left(\frac{\sigma^{2}}{2}-\frac{z^{2}}{2}\right) \nabla(\nabla \cdot(\vec{u}))
\end{gathered}
$$

that represents the second order term in power series expansion of the velocity vector about $\sigma$, in which $\nabla$ is the two-dimensional differential operator defined as $\nabla=\left(\frac{\partial}{\partial x}, \frac{\partial}{\partial y}\right)$ in a Cartesian reference system. We define $\overrightarrow{\vec{v}}$ as the depth averaged value of $\vec{v}(z)$, obtained by retaining terms of order $O\left(\mu^{2}, \varepsilon^{2} \mu^{2}\right)$, which is

$$
\begin{gathered}
\overrightarrow{\bar{v}}=\frac{1}{H} \int_{-h}^{\eta} \vec{v}(z) d z= \\
\left(\frac{\sigma^{2}}{2}-\frac{1}{6}\left(h^{2}-h \eta+\eta^{2}\right)\right) \nabla(\nabla \cdot(\vec{u})) \\
+\left(\sigma+\frac{1}{2}(h-\eta)\right) \nabla(\nabla \cdot(h \vec{u}))
\end{gathered}
$$


We define $x^{l}=x^{l}\left(\xi^{1}, \xi^{2}\right)$ (with $\left.l=1,2\right)$ as the transformation from the Cartesian coordinate system $\vec{x}$ to the curvilinear coordinate system $\vec{\xi}$ (henceforth the superscript indicates components and not powers). Let $\vec{g}_{(l)}=\partial \vec{x} / \partial \xi^{l}$ be the covariant base vector and $\vec{g}^{(l)}=\partial \xi^{l} / \partial \vec{x}$ be the contravariant base vector. The metric tensor and its inverse are given respectively by $g_{l m}=\vec{g}_{(l)} \cdot \vec{g}_{(m)}$ and $g^{l m}=\vec{g}^{(l)}$. $\vec{g}^{(m)}$. The Jacobian of the transformation is $\sqrt{g}=$ $\sqrt{\operatorname{det}\left(g_{l m}\right)}$. The transformation relationships between the components of the generic vector $\vec{b}$ in the Cartesian coordinate system and its contravariant and covariant components, $b^{l}$ and $b_{l}$, in the curvilinear coordinate system are given in the Appendix.

In order to apply a shock capturing scheme to the Boussinesq type equations, the convective terms must be expressed in conservative form, i.e. in divergence form. In [4] the system evolution variables were the conserved variables $H$ e $H u^{l}$. The choice of these conserved variables implied the presence of a source term in the mass conservation equation (right-hand side of Equation (4) in [4]. In this paper we choose, as conserved variables, the total local depth $H$ and the contravariant quantity

$$
M^{l}=H\left(u^{l}+\bar{v}^{l}\right)
$$

in which $\left(u^{l}+\bar{v}^{l}\right)$ represents the depth averaged horizontal velocity. With this choice, considering that the bottom depth does not vary over time, the contravariant integral form of the continuity Equation can be written as

$$
\iint_{\Delta A} \frac{\partial H}{\partial t} d A+\int_{L} M^{m} n_{m} d L=0
$$

where $L$ is the contour line of the surface element of area $\Delta A$ and $n_{m}$ is the m-th component of the covariant outward normal. As follows from Equation (5), the choice of the conserved variable $M^{l}$ expressed by Equation (4) makes it possible to write the continuity equation without any source term, but only with a flux term (second term of Equation 5).

This result implies that the continuity equation can be solved entirely by a high order shock capturing finite volume scheme.

With this new conserved variable, the integral over the surface element of area $\Delta A$ of the contravariant momentum equation can be written as

$$
\begin{gathered}
\iint_{\Delta A} \frac{\partial M^{1}}{\partial t} d A+\iint_{\Delta A}\left(\frac{M^{l} M^{m}}{H}\right)_{, m} d A \\
=-\iint_{\Delta A} G H g^{l m} \eta_{, m} d A \\
-\iint_{\Delta A} H\left(V^{l}+T^{l}+W^{l}+R^{l}\right) d A
\end{gathered}
$$

$$
\begin{gathered}
-\iint_{\Delta A} H\left(\frac{\partial \bar{v}^{l}}{\partial t}+\bar{v}_{, m}^{l} u^{m}+\right. \\
\left.u_{, m}^{l} \bar{v}^{m}+\bar{v}_{, m}^{l} \bar{v}^{m}\right) d A
\end{gathered}
$$

in which $u^{l}$ and $\bar{v}^{l}$ are the contravariant components of the vectors $\vec{u}$ and $\vec{v} ; \mathrm{G}$ is acceleration due to gravity. $R^{l}, V^{l}, T^{l}, W^{l}$ are, respectively, the contravariant components bottom resistance term, dispersive terms obtained by retaining terms of order $O\left(\mu^{2}, \varepsilon^{3} \mu^{2}\right)$ and term related to the second order approximation of the vertical vorticity expressed by

$$
\begin{gathered}
V^{l}=\frac{\sigma^{\wedge}}{2} g^{l m}\left[\left(\frac{\partial u^{k}}{\partial t}\right)_{, k}\right]_{, m} \\
+\sigma g^{l m}\left[\left(h \frac{\partial u^{k}}{\partial t}\right)_{, k}\right]_{, m} \\
-g^{l m}\left[\frac{1}{2} \eta^{\wedge}\left(\frac{\partial u^{k}}{\partial t}\right)_{, k}+\eta\left(h \frac{\partial u^{k}}{\partial t}\right)_{, k}\right]_{, m} \\
T^{l}=g^{l m}\left\{(\sigma-\eta) u^{i}\left(\left[h u^{k}\right]_{, k}\right)_{, i}\right. \\
\left.+\frac{1}{2}\left(\sigma^{\wedge}-\eta^{\wedge}\right) u^{i}\left(u_{, k}^{k}\right)_{, i}\right\}_{, m} \\
+\frac{1}{2} g^{l m}\left\{\left[\left(h u^{k}\right)_{, k}+\eta u_{, k}^{k}\right]^{\wedge}\right\}_{, m} \\
W^{l}=\left(\varepsilon^{m i} g_{i p} u_{, m}^{p}\right) \varepsilon^{j l} \bar{v}_{j} \\
+\left(\varepsilon^{m i} g_{i p} \bar{v}_{, m}^{p}\right) \varepsilon^{j l} u_{j}
\end{gathered}
$$

in which

$$
\begin{gathered}
\varepsilon^{m i}= \\
\left\{\begin{array}{c}
\frac{1}{\sqrt{g}} i f(m, i) \text { is a even permutation of }(1,2) \\
-\frac{1}{\sqrt{g}} \text { if }(m, i) \text { is a odd permutation of }(1,2) \\
0 \text { if the indexes are equal }
\end{array}\right.
\end{gathered}
$$

The "^" symbol indicates the operation of power raising and $\eta_{c}$ is an arbitrary constant value.

Motion equations admit stationary solutions in which the source terms are perfectly balanced by the flux terms. A numerical scheme is said to be wellbalanced and satisfies the C-Property if it preserves the steady state solutions exactly. The surface gradient term could be split into a source term that is related to the bed slope and a term related to the gradient of the square of the local total depth, in order to include this term into the flux term and to perform a shock capturing upwind scheme.

In particular, in the absence of motion, the numerical discretization of the source term relative to the bottom slope should perfectly balance the numerical discretization of the term related to the gradient of the square of the total local depth. Shi et 
al [5] point out that the above decomposition induces a numerical imbalance problem and does not allow the numerical scheme to satisfy the C-Property for non-uniform bed.

In order to obtain a "well-balanced" numerical scheme, the surface gradient term is decomposed as

$$
\begin{gathered}
G g^{l m} H \eta_{, m}=\left(G g^{l m} \frac{H^{\wedge} 2}{2}\right)_{{ }_{m}} \\
-G\left(\eta-\eta_{c}\right)\left(g^{l m} h\right)_{, m}-G \eta_{c}\left(g^{l m} h\right)_{, m} \\
-G\left(g^{l m} \frac{h^{\wedge} 2}{2}\right)_{, m}
\end{gathered}
$$

We split the term $V^{l}$ on the right-hand side of Equation (6) as follows

$$
V^{l}=\frac{\partial V^{\prime l}}{\partial t}+V^{\prime \prime l}
$$

in which $V^{\prime l}$ and $V^{\prime \prime l}$ are expressed by

$$
\begin{gathered}
V^{\prime l}=\frac{1}{2} \sigma^{\wedge} g^{l m}\left[\left(u^{k}\right)_{, k}\right]_{, m} \\
+\sigma g^{l m}\left[\left(h u^{k}\right)_{, k}\right]_{, m} \\
-g^{l m}\left[\frac{1}{2} \eta^{\wedge}\left(u^{k}\right)_{, k}+\eta\left(h u^{k}\right)_{, k}\right]_{, m} \\
V^{\prime \prime l}=g^{l m}\left[\frac{\partial}{\partial t}\left(\frac{\eta^{\wedge}}{2}\right)\left(u^{k}\right)_{, k}\right]_{, m} \\
+g^{l m}\left[\frac{\partial \eta}{\partial t}\left(h u^{k}\right)_{, k}\right]_{, m}
\end{gathered}
$$

We define with $D^{l}$ an auxiliary variable defined by

$$
D^{l}=H\left(u^{l}+V^{\prime l}\right)
$$

In order to obtain a contravariant integral form of the Fully Nonlinear Boussinesq Equations devoid of Christoffel symbols, we identify a physical direction with the one of the contravariant base vector $\overrightarrow{\tilde{g}}^{(l)}$ which is defined at the centre of $\Delta \mathrm{A}$. We take the projection, in the direction of vector $\overrightarrow{\tilde{g}}^{(l)}$, of the rate of change of the momentum of the material volume of fluid that at the generic instant $t$ coincides with $\Delta \mathrm{A}$. We equate this projection to the projection, in the same direction, of the net force acting on the material volume. By substituting Equations (11), (12) and (15) into Equation (6), and by adopting the abovementioned procedure we obtain

$$
\begin{gathered}
\frac{\partial \widetilde{D}^{l}}{\partial t}= \\
\frac{1}{\Delta A}\left\{-\sum_{\mu=1}^{2}\left[\int _ { \Delta \xi ^ { \mu + } } \left(\overrightarrow{\tilde{g}}^{(l)} \cdot \vec{g}_{(k)} \frac{M^{k} M^{\mu}}{H}+\right.\right.\right. \\
\left.\overrightarrow{\tilde{g}}^{(l)} \cdot \vec{g}^{(\mu)} G \frac{H^{\wedge}}{2}\right) \sqrt{g} d \xi^{\nu}-
\end{gathered}
$$

$$
\begin{aligned}
& \int_{\Delta \xi^{\mu-}}\left(\overrightarrow{\tilde{g}}^{(l)} \cdot \vec{g}_{(k)} \frac{M^{k} M^{\mu}}{H}+\right. \\
& \left.\left.\overrightarrow{\tilde{g}}^{(l)} \cdot \vec{g}^{(\mu)} G \frac{H^{\wedge}}{2}\right) \sqrt{g} d \xi^{\nu}\right]+ \\
& \iint_{\Delta A} G\left(\eta-\eta_{c}\right) \overrightarrow{\tilde{g}}^{(l)} \text {. } \\
& \vec{g}_{(k)} g^{k m} h_{, m} \sqrt{g} d \xi^{1} d \xi^{2}+ \\
& G \eta_{c} \sum_{\mu=1}^{2}\left[\int_{\Delta \xi^{\mu+}} \overrightarrow{\tilde{g}}^{(l)} \cdot \vec{g}^{(\mu)} h \sqrt{g} d \xi^{\nu_{-}}\right. \\
& \left.-\int_{\Delta \xi^{\mu-}} \overrightarrow{\tilde{g}}^{(l)} \cdot \vec{g}^{(\mu)} h \sqrt{g} d \xi^{\nu}\right]+ \\
& \frac{\mathrm{G}}{2} \sum_{\mu=1}^{2}\left[\int_{\Delta \xi^{\mu+}} \overrightarrow{\tilde{g}}^{(l)} \cdot \vec{g}^{(\mu)} h^{\wedge} \sqrt{g} d \xi^{\nu}-\right. \\
& \left.\int_{\Delta \xi^{\mu-}} \overrightarrow{\tilde{g}}^{(l)} \cdot \vec{g}^{(\mu)} h^{\wedge} \sqrt{g} d \xi^{\nu}\right]- \\
& \iint_{\Delta A} H \overrightarrow{\tilde{g}}^{(l)} \cdot \vec{g}_{(k)} V^{\prime \prime k} \sqrt{g} d \xi^{1} d \xi^{2}- \\
& \iint_{\Delta A} H \overrightarrow{\tilde{g}}^{(l)} \cdot \vec{g}_{(k)}\left(T^{k}+W^{k}+\right. \\
& \left.R^{k}\right) \sqrt{g} d \xi^{1} d \xi^{2}+ \\
& \iint_{\Delta A} \overrightarrow{\tilde{g}}^{(l)} \cdot \vec{g}_{(k)} \frac{\partial H}{\partial t}\left(V^{\prime k}-\bar{v}^{k}\right) \sqrt{g} d \xi^{1} d \xi^{2}+ \\
& \iint_{\Delta A} H\left(\overrightarrow{\tilde{g}}^{(l)} \cdot \vec{g}_{(k)} \bar{v}^{k}\right)_{, m} u^{m} \sqrt{g} d \xi^{1} d \xi^{2}+ \\
& \iint_{\Delta A} H\left(\overrightarrow{\tilde{g}}^{(l)} \cdot \vec{g}_{(k)} u^{k}\right)_{, m} \bar{v}^{m} \sqrt{g} d \xi^{1} d \xi^{2}+ \\
& \left.\iint_{\Delta A} H\left(\overrightarrow{\tilde{g}}^{(l)} \cdot \vec{g}_{(k)} \bar{v}^{k}\right)_{, m} \bar{v}^{m} \sqrt{g} d \xi^{1} d \xi^{2}\right\}
\end{aligned}
$$

where $\widetilde{D}^{l}$ represent the averaged value of $D^{l}$ over the surface element of area $\Delta \mathrm{A}$, defined as

$$
\widetilde{D}^{l}=\frac{1}{\Delta A} \iint_{\Delta A} D^{l} \sqrt{g} d \xi^{1} d \xi^{2}
$$

Over the same element of area $\Delta \mathrm{A}$, the integral form of the continuity Equation (6) reads

$$
\begin{gathered}
\frac{\partial \widetilde{H}}{\partial t}=-\frac{1}{\Delta A} \sum_{\mu=1}^{2}\left[\int_{\Delta \xi^{\mu+}} M^{\mu} \sqrt{g} d \xi^{\nu-}\right. \\
\left.\int_{\Delta \xi^{\mu-}} M^{\mu} \sqrt{g} d \xi^{\nu}\right]
\end{gathered}
$$

where $\widetilde{H}$ represents the average value of $H$ over the surface element of area $\Delta \mathrm{A}$

$$
\widetilde{H}=\frac{1}{\Delta A} \iint_{\Delta A} H \sqrt{g} d \xi^{1} d \xi^{2}
$$

Equations (16) and (18) represent a new integral form of the Fully Nonlinear Boussinesq Equations expressed in a contravariant formulation in which Christoffel symbols are absent. These equations are accurate to $O\left(\mu^{2}, \varepsilon^{3} \mu^{2}\right)$ in dispersive terms and conserve vertical vorticity with a leading-order error of $O\left(\mu^{4}\right)$. In the above-mentioned equations the conserved variables are $H$ and $M^{l}$. Consequently, the momentum balance equation differs from the one presented by Cannata et al. [4] for the different expression of the convective terms. Furthermore, unlike the [4] model, in the continuity equation no dispersive term is present. This result makes it 
possible to solve the continuity equation entirely by a high order shock capturing finite volume scheme. In this way in the numerical solution the errors due to the discretization of the dispersive term in the continuity equation are not introduced. Furthermore, the surface gradient term has been split in order to solve this term by a finite volume technique and to obtain a "well balanced" numerical scheme.

In order to simulate the undertow Lynett [7] proposed a correction to the vertical distribution of the horizontal velocity calculated by depth-averaged motion equations. Indicating by $u_{B}^{k}(z)$ the corrective contravariant velocity vector, the horizontal velocity $u^{k}(z)$ reads as follows

$$
\begin{aligned}
& u^{k}(z, t)=u_{\alpha}^{k}+(\sigma-z) g^{k r}\left[\left(h u_{\alpha}^{l}\right)_{, l}\right]_{, r}+ \\
& {\left[\left(\sigma^{2} / 2\right)-\left(z^{2} / 2\right)\right] g^{k r}\left[\left(u_{\alpha}^{l}\right)_{, l}\right]_{, r}+u_{B}^{k}(z)}
\end{aligned}
$$

where $u_{\alpha}^{k}$ is the horizontal velocity contravariant vector computed by Equations (16) and (18). We indicate by $U(z, t)$ the Cartesian horizontal component of the fluid velocity; $\Omega(t)$ indicates the thickness of the boundary layer, $U_{\Omega}(t)$ the horizontal velocity at the top of the wave boundary layer and $u_{f}(t)$ the friction velocity. From the integration of the momentum balance equation inside the boundary layer gives and from the logarithmic law of the velocity profile, we obtain the equation

$$
\begin{gathered}
-u_{f}^{2}(t)=-\Omega(t) \frac{d U_{\Omega}(t)}{d t}+ \\
\frac{1}{K} \frac{d u_{f}(t)}{d t} \frac{k}{30}\left[e^{\frac{U_{\Omega}(t)}{u_{f}(t)} K}\left(\frac{U_{\Omega}(t)}{u_{f}(t)} K-1\right)+1\right]
\end{gathered}
$$

where $k / 30$, represents the characteristic length scale, in which $k$ is the bed roughness and $K$ is the von Karman constant. The thickness of the boundary layer is obtained by the following equation

$$
\Omega(t)=\left(e^{\frac{U_{\Omega}(t)}{u_{f}(t)} K}-1\right) \frac{k}{30}
$$

Solving the system composed by Equations (21) and (22) the values of $u_{f}(t)$ and $\Omega(t)$ are given. The average of the instantaneous quantities over the wave period $T$ is indicated by the mark [ $]$. The turbulence inside the boundary layer is produced by the interaction between wave and current. Let be $\widetilde{\mathrm{u}_{\mathrm{fc}}}$ the current friction velocity given by

$$
{\widetilde{u_{f c}}}^{2}=\frac{1}{T} \int_{0}^{T} u_{f}^{2}(t) d t
$$

Within the boundary layer, the eddy viscosity is

$$
\begin{gathered}
v_{t, r}(z, t)= \\
K u_{f}(t) z\left[1-\frac{z}{\Omega(t)}\left(1-\frac{\widetilde{u_{f c}}}{u_{f}(t)}\right)\right]\left(1-\frac{z}{\widetilde{H}}\right)
\end{gathered}
$$

While, outside the boundary layer the eddy viscosity is

$$
v_{t, r}(z)=\widetilde{u_{f c}} K z\left(1-\frac{z}{\widetilde{H}}\right)
$$

Under breaking waves, the turbulence is given by the contributions produced by current, wave boundary layer and wave breaking. The turbulent kinetic energy equation [14] comes into in the calculation of the eddy viscosity $v_{t, f}(z, t)$ related to the breaking of the wave

$$
\frac{\partial k_{t}}{\partial t}=\frac{P(k)}{\rho}-c_{d} \frac{k_{t}^{\frac{3}{2}}}{l}+\frac{\partial}{\partial z}\left(v_{t, f}(z, t) \frac{\partial k_{t}}{\partial z}\right)
$$

where $l$ is the turbulence length scale, $k_{t}=k_{t}(z, t)$ is the kinetic energy of the wave breaking-induced turbulence and $c_{d}=0.08$. The kinetic energy production $P(k)$ is calculated according to [14].

The integration of Equation (26) gives the instantaneous value of $k_{t}$ which is used in order to calculate the eddy viscosity produced by the wave breaking as

$$
v_{t, f}(z, t)=l \sqrt{k_{t}}
$$

Consequently, the total eddy viscosity $v_{t}(z, t)$ is the quadratic sum of the eddy viscosity due to the current and wave breaking and the eddy viscosity produced by the wave boundary layer

$$
v_{t}^{2}(z, t)=v_{t, r}^{2}(z, t)+v_{t, f^{2}}^{2}(z, t)
$$

The instantaneous values of $u^{k}, u_{f}, v_{t}, H$ and $\Omega$ are used to calculate the input variables of the morphodynamic model, as shown in the following Subsection.

\subsection{Morphodynamic model}

The integral contravariant form of the solid particle concentration equation, in which a quasi-threedimensional methodology is used, is expressed by

$$
\begin{aligned}
& \iint_{\Delta A} \frac{\partial \tilde{\tilde{C}} \widetilde{H}}{\partial t} d A+\int_{L}\left[\int_{a}^{\widetilde{H}} \tilde{C}(z) \tilde{u}^{r}(z) d z\right] n_{r} d L- \\
& \int_{L} \overline{\widetilde{v_{t}}} \widetilde{H} b^{r k}(\overline{\tilde{C}})_{, k} n_{r} d L=\iint_{\Delta A}(P-D) d A
\end{aligned}
$$

in which $\vec{n}$ is the outward normal vector to the contour line $L ; \tilde{C}(z)$ is the solid particle concentration averaged on the wave period; $\widetilde{H}$ is the water depth; $\tilde{u}^{r}$ is the horizontal contravariant velocity vector averaged over the wave period; $a$ is the distance from the bottom which defines the region in which the bed load transport develops; $\overline{\widetilde{v_{t}}}$ is the depth and waveaveraged eddy viscosity; $D$ is the rate of the sediment deposition and $P$ is the rate of turbulent sediment pick-up. $D$ and $P$ are expressed by 


$$
\begin{aligned}
& D=w_{\text {sed }} \tilde{C}_{a} \\
& P=w_{\text {sed }} \tilde{C}_{R}
\end{aligned}
$$

in which $w_{\text {sed }}$ is the sediment fall velocity, $\tilde{C}_{a}$ and $\tilde{C}_{R}$ are, respectively, the actual and reference concentrations, that are evaluated at height $a=2 d_{50}$. A threshold value of the sediment particle motion comes into the calculation of $\tilde{C}_{R}$. In order to integrate Equation (29), the calculation of $\tilde{C}_{a}$ and $\tilde{C}_{R}$ is needed and shown hereinafter. The value of $\tilde{C}_{a}$ is taken as the lower boundary condition of the following turbulent suspended sediment diffusion equation

$$
-\tilde{C}(z) w_{s e d}=\widetilde{v_{t}}(z) \frac{\partial \tilde{C}(z)}{\partial z}
$$

and as the lower extreme of the integral that gives the depth-averaged value of $\tilde{C}(z)$

$$
\overline{\tilde{C}}=\frac{1}{\widetilde{H}} \int_{a}^{\widetilde{H}} \tilde{C}(z) d z
$$

Thus, $\tilde{C}_{a}$ is calculated by an iterative procedure using Equation (33), where the values of $\overline{\tilde{C}}$ and $\widetilde{v_{t}}(z)$ are known (from the previous time step). The value of $\tilde{C}_{R}$ is obtained by wave-averaging its instantaneous values $C_{R}(t)$ that are calculated according to [15]. The total transport is given by the sum of the bed load transport, which takes into account the near bed transport mechanism, and the suspended load transport. The contravariant equation of the bed change expressed in a curvilinear coordinate system is

$$
\frac{\partial z_{f}}{\partial t}=-\frac{1}{1-p}\left[(P-D)+\tilde{q}_{b, k}^{k}\right]
$$

in which $p$ is the porosity of the sediment and $z_{f}$ is the elevation of the bed; $\tilde{q}_{b}^{k}(k=1,2)$ is the contravariant components of the bed load transport vector $\widetilde{\vec{q}}_{b}$ that is define as

$$
\begin{array}{r}
\tilde{\overrightarrow{\vec{q}}}_{b}=\frac{1}{T} \int_{0}^{T}\left(5\left(1+\frac{\frac{\pi}{6} \beta}{|\vec{\theta}|-\theta_{c r}}\right)^{-1 / 4}(\sqrt{|\vec{\theta}|}-\right. \\
\left.\left.\quad 0.7 \sqrt{\theta_{c r}}\right) \sqrt{\left(\frac{\rho_{s}}{\rho_{w}}-1\right) G d_{50}^{3}}\right) \frac{\vec{u}_{a}}{\left|\vec{u}_{a}\right|} d t
\end{array}
$$

in which $\beta$ is the coefficient of the dynamic friction; $\rho_{s} / \rho_{w}$ is the ratio between the sediment and water density; $\theta_{c r}$ is the parameter of stability of Shield and $|\vec{\theta}|=|\vec{\theta}(t)|$ is the parameter of mobility of Shield, where $\vec{\theta}(t)$ is the bed shear stress induced by current and wave and $d_{50}$ is the sediment mean diameter.

\section{Sandbar formation}

In this Section, we numerically reproduce an experiment extracted from "LIP 11D Delta Flume Experiments", described in the data report by Roelvink and Reniers [16]. The report contains hydrodynamic and sediment transport measures. The experiments was conducted in a wave flume with a $183 \mathrm{~m}$ long mobile bottom and were carried out so as to reproduce slightly erosive wave conditions acting for $12 h$. In this test, narrow-banded random waves was generated (by a random phase, linear generator from a JONSWAP spectrum) at $x=0 m$ normally incident to the coast, whose characteristics are shown in Table 1. The bottom profile of the wave flume used as initial condition and the still water level are shown in Fig. 1. The wave flume was characterized by three different regions: a first region in which the depth is equal to $4.1 \mathrm{~m}$; a second region in which the water depth varies following a Dean-type bottom profile (the so-called equilibrium beach parabolic profile of Brunn-Dean-More); a third region in which the water depth varies following a constant bottom slope. The initial position of the shoreline is approximately located at $x=181 \mathrm{~m}$. In the experiment, the sediment was characterized by a mean diameter of $220 \mu \mathrm{m}$.

Table 1. Incident wave characteristics

\begin{tabular}{cccc}
\hline$H_{0}(\mathbf{m})$ & $T_{p}(\mathrm{~s})$ & $\begin{array}{c}\text { Water level } \\
(\mathbf{m})\end{array}$ & Duration $(\mathbf{h})$ \\
\hline 0.9 & 5 & 4.1 & 12 \\
\hline
\end{tabular}

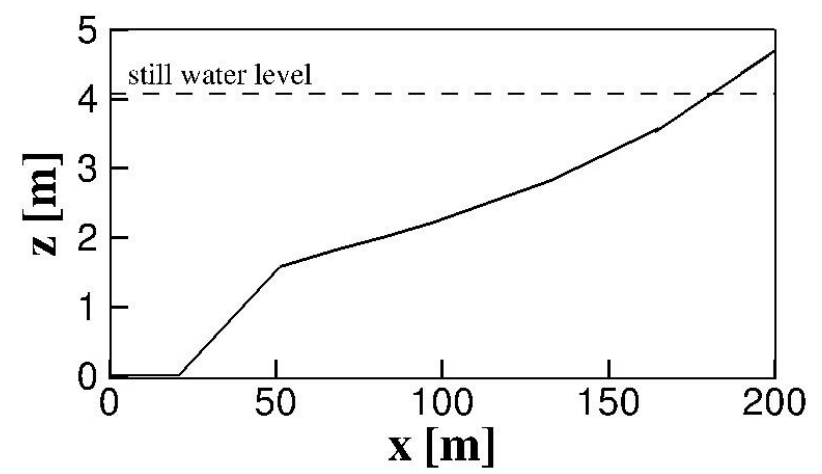

Fig. 1. Initial bottom profile (solid line) and still water level (dashed line)

The test is numerically reproduced by internally generating random wave trains, characterized by a JONSWAP frequency spectrum with a significant wave height respectively of $0.9 \mathrm{~m}$ and $1.4 \mathrm{~m}$. The wave-averaged total sediment transport calculated by using the proposed model and compared with respect to the experimental measurements is shown in Fig. 2.

The sediment resuspension phenomena are larger in the first region, located around at $x=145 \mathrm{~m}$, where the wave breaking starts. Fig. 2 also shows that in the breaking zone, the wave-averaged total sediment transport is offshore directed. The waveaveraged total sediment transport in the second 
region, located around at $x=170 \mathrm{~m}$, is represented by the sediment contribution provided by the swash zone. The wave-averaged total sediment transport is

offshore directed even in this region.

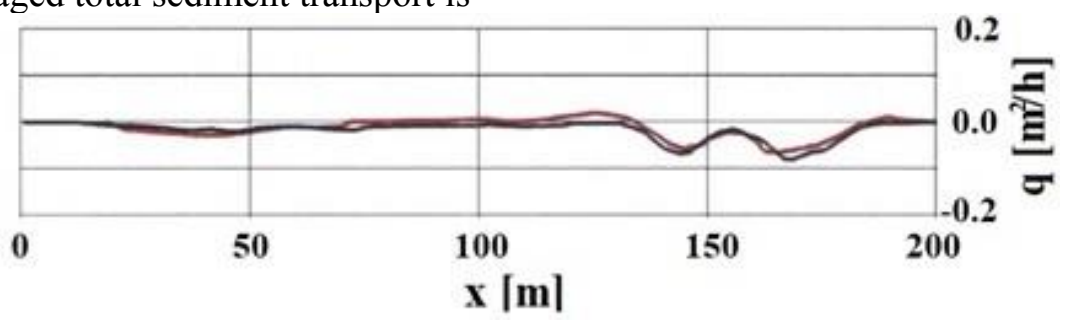

Fig. 2. Comparison of calculated (black line) and measured (red line) wave-averaged total sediment transport by using the proposed model.

Is to be underlined that, in the proposed model, the suspended sediment transport contribution to the wave-averaged total sediment transport is higher than the bed load transport contribution. Indeed, in the proposed model, the separation point between the region in which the suspended sediment transport is dominant and the region in which the bed load transport is dominant, is placed nearer than $2.5 d_{50}$ from the bed. In fact, following [17], the thickness of the region in which the bed load transport is dominant (i.e. by the sediment particles moving by rolling, sliding, or in short jumps) is particularly small.

The sediment particles, in the upper region, are held in suspension by the turbulence. The strong turbulence induced by waves, since the sediment consists of very fine sand, tends to put into suspension most of the sediment and therefore the bed load transport is small.

The numerical results are in good agreement with the experimental measurements. The numerical results show that model is able to capture the sediment resuspension phenomena produced by wave breaking and the offshore sediment transport due to undertow currents.

Fig. 3 shows the comparison between the numerical and experimental results, in terms of bed modifications. From Fig. 3 it can be seen that two sandbars in the nearshore zone are present. The first sandbar is formed in the breaker zone. The second sandbar is formed in the nearer area to the swash zone.

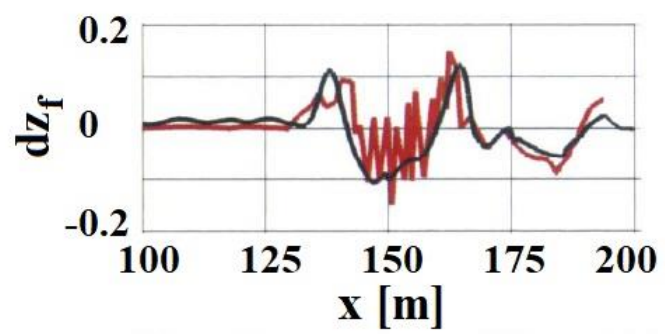

Fig. 3. Comparison of calculated (black line) and measured (red line) bed modifications by using the proposed model.
The first sandbar is located around at $x=137 \mathrm{~m}$ and is characterized by a crest height of $0.1 \mathrm{~m}$ about. The above-mentioned sandbar is produced by the deposit of sediments put into suspension near the wave breaking $(145 m<x<155 m)$ and transported by undertow currents. In fact, the suspended sediment is bound to settle just seaward of the breaker zone due to a strong turbulence reduction. The second sandbar is located around at $x=165 \mathrm{~m}$, it is characterized by a crest height of about $0.12 \mathrm{~m}$ and it is produced by contribution of the transported material from the swash zone.

A similar seaward sandbar migration dynamic involves the movement of the second sandbar located in the nearer area to the swash zone. The numerical results are in good agreement with the experimental measurements. The bar migration and the general trends of the beach profile changes are well predicted by the proposed model.

\section{Sea bottom modifications behind a T-head groin}

In this Section, we numerically reproduce a Test T3C1 extracted from "LSTF Experiments Transport by Waves and Currents \& Tombolo Development Behind Headland Structures", described in the data report by Gravens and Wang [18]. That Test was carried out experimentally on a natural beach with a 4-m long T-head groin centrally located in the alongshore direction of the model beach and with head section parallel-positioned $4 \mathrm{~m}$ offshore of the initial shoreline. It had a duration of $184 \mathrm{~min}$. A random wave was generated by a waves generator and it was characterized by a $0.26 \mathrm{~m}$ significant breaking wave height, $1.5 \mathrm{~s}$ period and an approximate wave angle of $6.5^{\circ}$ with respect to the shoreline. In the experiment, the sediment were characterized by a mean diameter of $150 \mu \mathrm{m}$. Test $\mathrm{T} 3 \mathrm{C} 1$ is numerically reproduced by internally generating random wave trains characterized by a 
JONSWAP frequency spectrum and a significant breaking wave height of $0.26 \mathrm{~m}$.

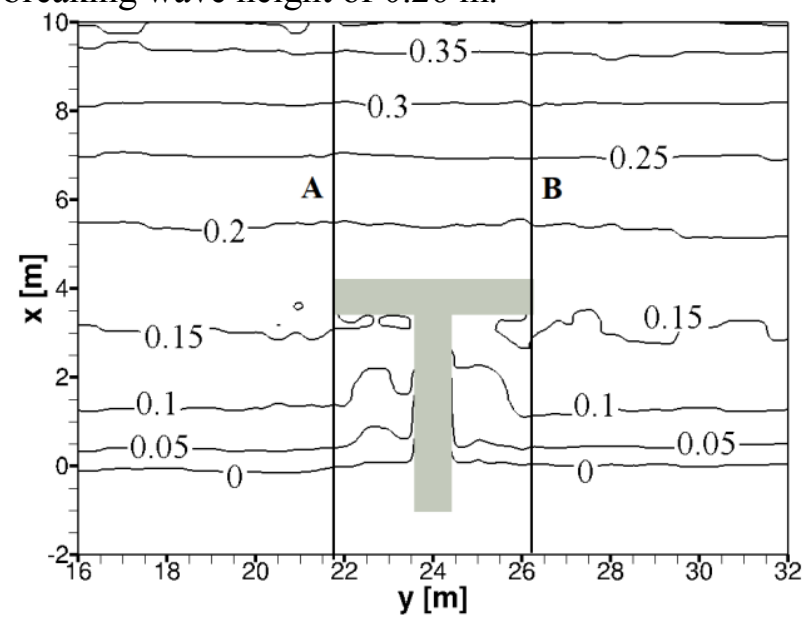

Fig. 4. Initial depth contour lines (black solid lines), and comparison sections (black solid line $\mathrm{A}$ and $\mathrm{B}$ ) for Test $\mathrm{T} 3 \mathrm{Cl}$.

Fig. 4 illustrates the depth contour lines in the initial condition for Test $\mathrm{T} 3 \mathrm{Cl}$, and the two sections $\mathrm{A}$ and $\mathrm{B}$ where experimental data are known.

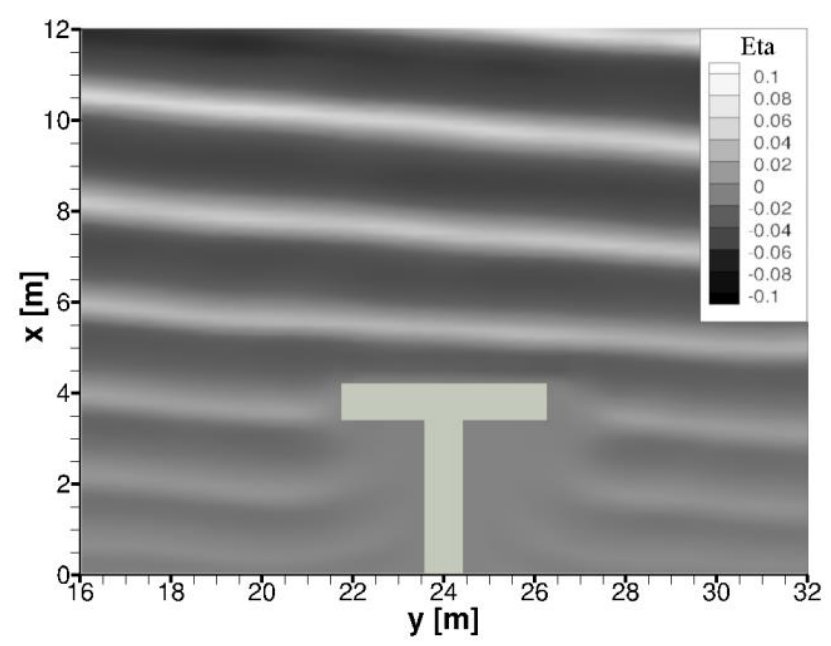

Fig. 5. Instantaneous wave field for Test T3Cl.

An instantaneous wave field obtained by the numerical simulation of Test $\mathrm{T} 3 \mathrm{C} 1$ carried out by the proposed model is shown in Fig. 5. It is observed that, starting from about $x=15 \mathrm{~m}$ toward the shoreline the wave height gradually decreases because of the breaking. In the same figure it is noted that the wave fronts, in the lee of the T-head groin, although attenuated by breaking undergo a rotation owing to the diffraction effects.

The wave-averaged velocity field obtained by the numerical simulation of Test $\mathrm{T} 3 \mathrm{C} 1$ carried out by the proposed model is compared with respect to the experimental measurements as shown in Fig. 6. It can be seen that the current is intercepted and offshore- diverted by the T-head groin. In the same figure, the simulated velocity field is characterized by the formation of two eddies close to the T-head groin. It is possible to notice that the wave-averaged velocity field obtained by numerical simulation is in good agreement with respect to the experimental measurements.

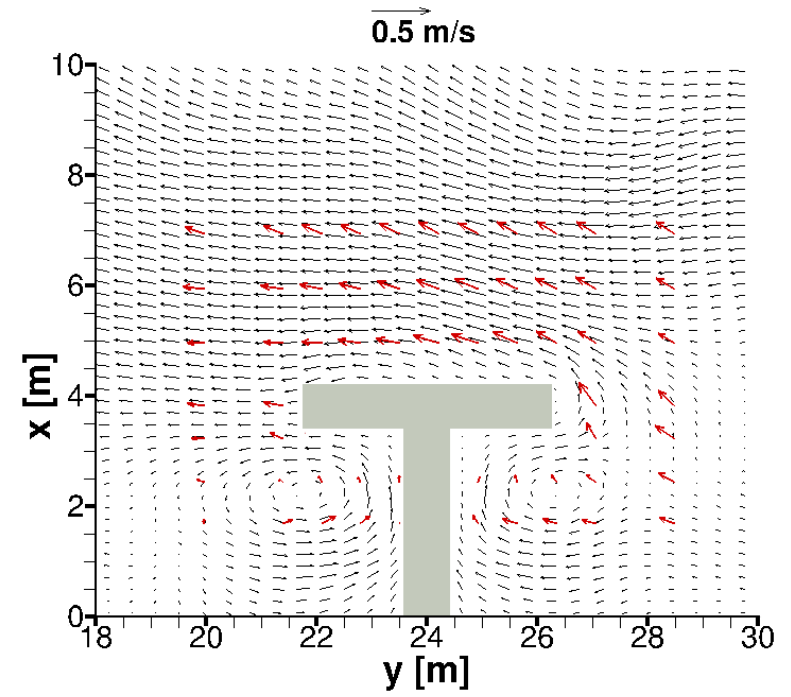

Fig. 6. Wave-averaged velocity field: calculated (black vectors) and measured (red vectors) velocity current for Test $\mathrm{T} 3 \mathrm{Cl}$.

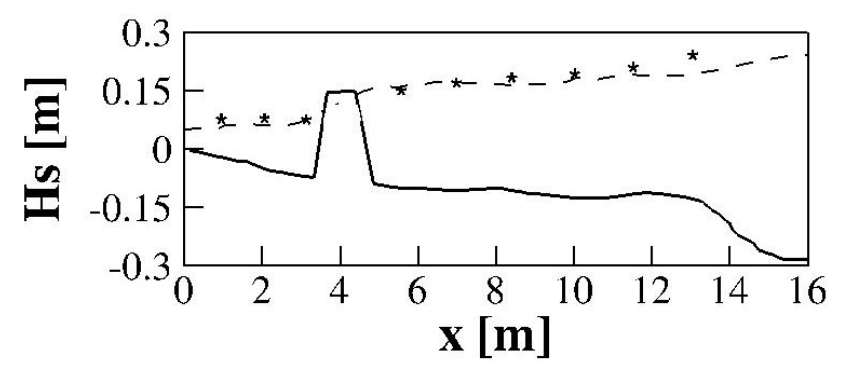

(a)

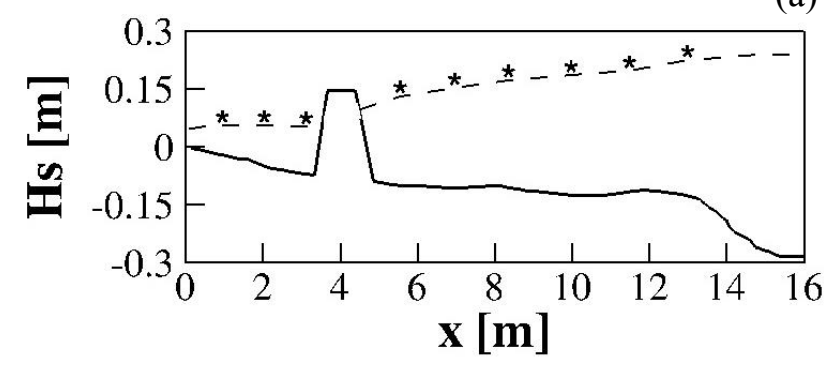

(b)

Fig. 7. Comparison of calculated (dashed line) and measured (asterisks) significant wave height for Test $\mathrm{T} 3 \mathrm{Cl}$ (beach profile in solid line) in section A (Fig. 7a) and in section B (Fig. 7b).

In Fig 7 the comparison between the numerical results and experimental measurements by Gravens and Wang [18], in terms of significant wave height, at the two sections $\left(y_{A}=26 \mathrm{~m}\right.$ and $\left.y_{B}=22 \mathrm{~m}\right)$ is shown. In Figs. 7(a) and 7(b) it is possible to notice 
that the sharp rise in the bed elevation induces the wave breaking and consequently the decay in wave height between $x=14 \mathrm{~m}$ and $x=12 \mathrm{~m}$. The wave height changes minimally in the zone from $x=12 \mathrm{~m}$ to $x=4 m$, being, in that zone, the bed elevation nearly constant. In the zone between the head section of the T-head groin and the shoreline, where the structure produces the maximum shielding effect on the incident waves, the wave height substantially decreases. These figures show that the numerical results are in good agreement with the experimental measurements.

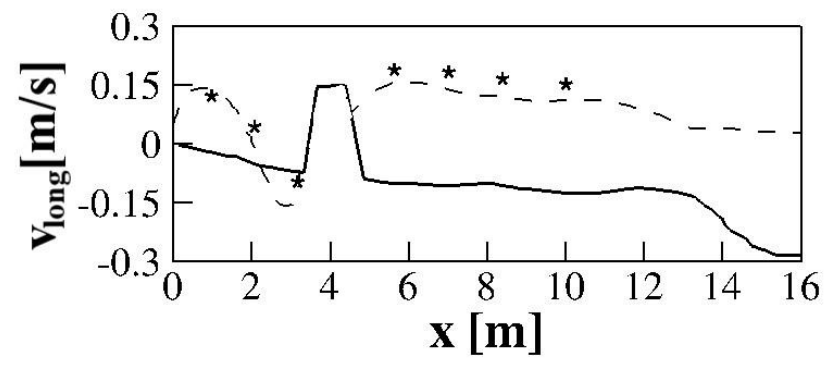

(a)

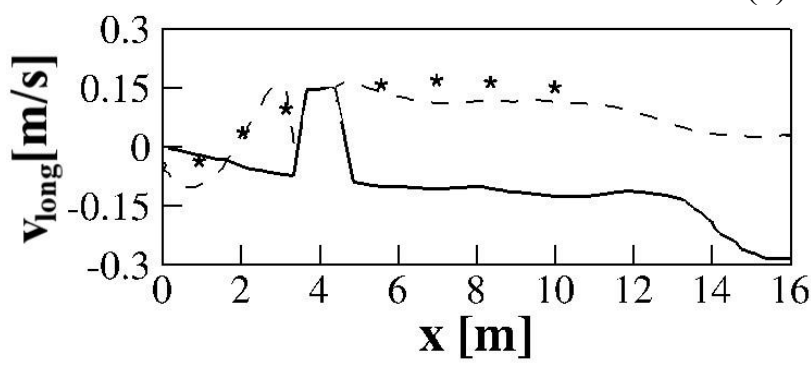

(b)

Fig. 8. Comparison of calculated (dashed line) and measured (asterisks) longshore current for Test T3C1 (beach profile in solid line) in section A (Fig. 8a) and in section B (Fig. 8b).

Fig. 8 shows the comparison between the longshore currents obtained by the numerical model proposed in this paper and the experimental measurements by Gravens and Wang [18] at the two sections previously indicated.

From Fig. 8a, it is possible to notice that, in the zone close to the head section downdrift side, $2 m<x<$ $4 m$, there is an inversion of the direction of the longshore current, due to the presence of a small eddy. In the zone from $x=2 m$ to $x=0 m$, the long-shore current is slightly overestimated.

The aforementioned inversion of the long-shore current is also present in section B (see Fig. 8b), due to the presence of a second eddy.

In these figures, it is observed that the numerical results are in good agreement with respect to the experimental measurements.

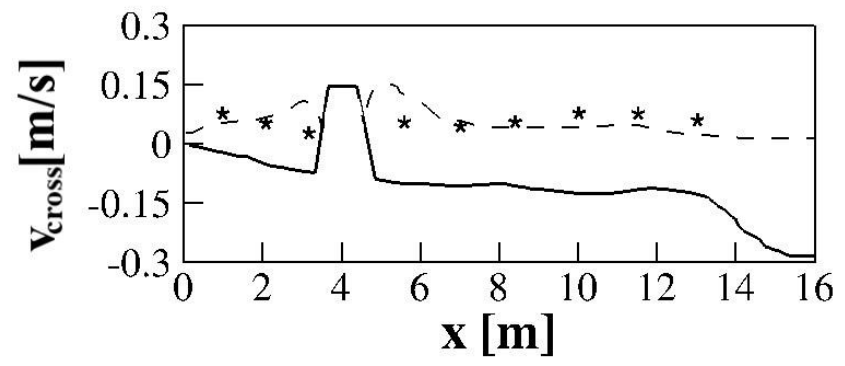

(a)

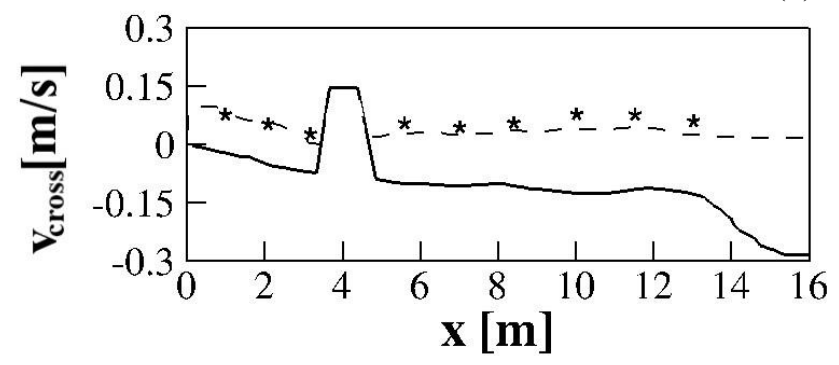

(b)

Fig. 9. Comparison of calculated (dashed line) and measured (asterisks) cross-shore current for Test $\mathrm{T} 3 \mathrm{Cl}$ (beach profile in solid line) in section A (Fig. 9a) and in section B (Fig. 9b).

Fig. 9 shows the comparison between the crossshore currents obtained by the numerical model proposed in this paper and the experimental measurements by Gravens and Wang [18] at the two sections previously described. In these figures, it is possible to notice that the numerical results are in good agreement with respect to the experimental measurements. In Fig. 10, the depth contour lines obtained at the end of the numerical simulation of Test $\mathrm{T} 3 \mathrm{C} 1$ carried out by the proposed model (black lines), and the corresponding depth contour lines obtained from experimental data by Gravens and Wang [18] (grey lines) are shown.

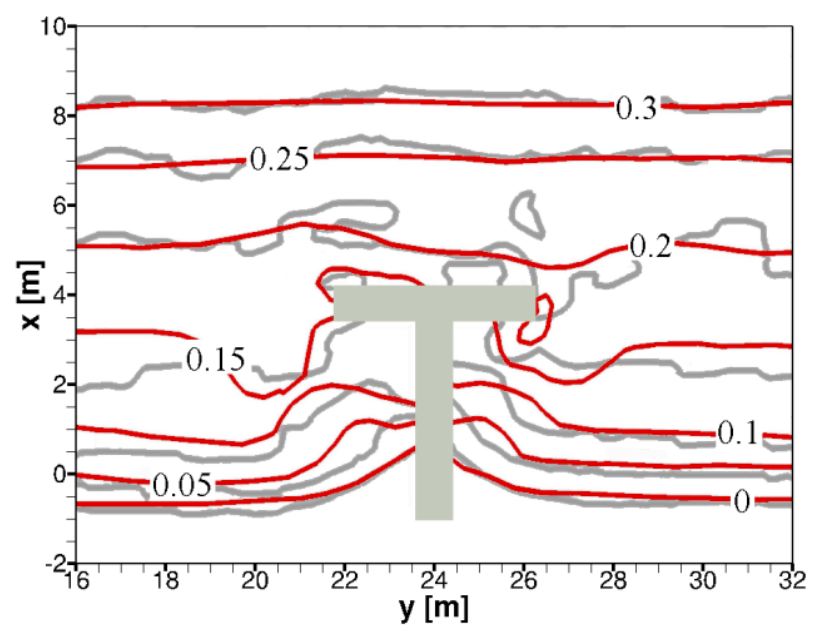

Fig. 10. Comparison of calculated (red lines) and measured (grey lines) depth contour lines for Test $\mathrm{T} 3 \mathrm{Cl}$. 
The comparison between Figs. 4 and 10 highlights the modifications in the bed elevation obtained by the numerical model. From this comparison it is possible to notice a seaward advancement of the shoreline development in the lee of the T-head groin and right near the stem: the advancement of the $0.0-0.1 \mathrm{~m}$ depth contour lines toward the head section is about $1 \mathrm{~m}$. The sediment puts into suspension in the region upstream of the T-head groin (with respect to longshore current direction) and the one coming from the swash zone produce an accretion in the updrift side of the stem: in the lee of the T-head groin, the accumulation of sediment is due to the decay of the current velocity highlighted by the presence of the above-mentioned first eddy. In the downdrift side of the stem, the rise in the bed elevation is produced by the sediment coming from the swash zone and transported by the second eddy toward the stem.

The comparison between Figs. 4 and 10 evidences also that, in proximity of the head section extremes, there are two erosion areas: the first one is located close to the top left corner of the T-head groin $(3.5 m<x<4.5 m$ and $26 m<y<28 m)$ where the depth contour line is $0.2 \mathrm{~m}$; the second one is located near the bottom right corner of the T-head groin $(2.5 m<x<3.5 m$ and $22 m<y<20.5 m)$ and is characterized by the retreat toward the shoreline of $0.15 \mathrm{~m}$ depth contour line.

In Fig. 10 the comparison between the measured depth contour lines obtained at the end of the experiment $\mathrm{T} 3 \mathrm{C} 1$ and the ones obtained by the proposed numerical model is shown. It can be noted a good agreement between the numerical and experimental results.

The numerical results show: a slight underestimation of the extension of the erosion area in the region near the shoreline located upstream of the T-head groin $(28 m<y<32 m)$; a small underestimation of the extension of the erosion area in the region near to the shoreline downstream of the T-head groin $(y>$ $21 \mathrm{~m}$ ); a slight underestimation of the extension of the erosion area near the top left corner of the T-head groin. The salient updrift and downdrift of the T-head groin stem is shown to be well predicted by the proposed numerical model.

The presence of the coastal defence structures induces a complex current velocity field that influences the local sediment transport. The wave induced longshore current transports the sediment, that are put into suspension by the breaking waves, up to the region upstream of the T-head groin; close to the stem of the T-head groin the current is offshore directed and the sediment are carried towards the lee of the T-head groin, where it settles.

\section{Conclusion}

In this paper, the modifications induced by a T-head groin on the sea bottom have been simulated by a new numerical model which is composed by a hydrodynamic model and a morphodynamic model. The good agreement between the numerical and experimental results shows the ability of the proposed hydrodynamic model, based on a new conservative integral contravariant form of the Fully Nonlinear Boussinesq Equations, and of the proposed morphodynamic model, based on the Quasi-Threedimensional approach, to simulate the sea bottom modifications behind a T-head groin.

\section{Appendix}

$$
\begin{gathered}
b^{l}=\vec{g}^{(l)} \cdot \vec{b} ; \quad b_{l}=\vec{g}_{(l)} \cdot \vec{b} \\
\vec{b}=b^{l} \vec{g}_{(l)} ; \quad \vec{b}=b_{l} \vec{g}^{(l)} \\
b^{l}{ }_{m}=\partial b^{l} / \partial \xi^{m}+\Gamma_{m k}^{l} b^{k} \\
\Gamma_{m k}^{l}=\vec{g}^{(l)} \cdot \partial \vec{g}_{(k)} / \partial \xi^{m} \\
T_{, m}^{l m}=\frac{1}{\sqrt{g}} \frac{\partial T^{l m} \sqrt{g}}{\partial \xi^{m}}+T^{n m} \Gamma_{n m}^{l}
\end{gathered}
$$

\section{References:}

[1] Cannata G., Petrelli C., Barsi L., Camilli F., Gallerano F., 3D free surface flow simulations based on the integral form of the equations of motion, WSEAS Transactions on Fluid Mechanics, Vol. 12, 2017, pp. 166-175.

[2] Cannata G., Gallerano F., Palleschi F., Petrelli C., Barsi L., Three-dimensional numerical simulation of the velocity fields induced by submerged breakwaters, International Journal of Mechanics, Vol. 13, 2019, pp. 1-14.

[3] Cannata G., Petrelli C., Barsi L., Gallerano F., Numerical integration of the contravariant integral form of the Navier-Stokes equations in time-dependent curvilinear coordinate systems for three-dimensional free surface flows, Continuum Mechanics and Thermodynamics, Vol. 31, No. 2, 2019, pp.491-519.

[4] Cannata G., Barsi L., Petrelli C., Gallerano F., Numerical investigation of wave fields and currents in a coastal engineering case study, WSEAS Transactions on Fluid Mechanics, Vol. 13, 2018, pp. 87-94.

[5] Shi F., Kirby J.T., Harris J.C., Geiman J.D., Grilli S.T., A high-order adaptive time stepping TVD solver for Boussinesq modelling of 
breaking waves and coastal inundation, Ocean Modelling, Vol.43-44, 2012, pp.35-51.

[6] Cannata G., Petrelli C., Barsi L., Fratello F., Gallerano F., A dam-break flood simulation model in curvilinear coordinates, WSEAS Transactions on Fluid Mechanics, Vol. 13, 2018, pp. 60-70.

[7] Lynett P.J., Nearshore wave modelling with high-order Boussinesq-type equations, Journal of Waterway, Port, Coastal, and Ocean Engineering, Vol.132(5), 2006, pp. 348-357.

[8] Wei G., Kirby J.T., Grilli S.T., Subramanya R., A fully non-linear Boussinesq model for surface waves. Part 1: Highly nonlinear unsteady waves. Journal of Fluid Mechanics, Vol.294, 1995, pp.71-92

[9] Nwogu O., Alternative form of Boussinesq equations for nearshore wave propagation, Journal of Waterway, Port, Coastal, and Ocean Engineering, Vol. 119, 1993, pp. 618-638.

[10] Chen Q., Kirby J.T., Dalrympe R.A., Shi F., Thornton E.B., Boussinesq modeling of longshore currents, Journal of Geophysical Research, Vol. 108, 2003, pp. 26-1;26-18.

[11] Chen Q., Fully non-linear Boussinesq-type equations for waves and currents over porous beds, Journal of Engineering Mechanics, Vol.132(2), 2006, pp. 220-230.

[12] Drønen N., Deigaard R., Quasi-threedimensional modelling of the morphology of longshore bars, Coastal Engineering, Vol.54, 2007, pp.197-215.

[13] Kim N.H., An S.H., Numerical computation of the nearshore current considering wave-current interactions a Gangjeong coastal area, Jeju Island, Korea, Engineering Applications of Computational Fluid Mechanics, Vol. 5, 2011, pp.430-444.

[14] Deigaard R., Fredsøe J., Hedegaard I.B., Suspendend sediment tin the surf zone, Journal of Waterway, Port, Coastal, and Ocean Engineering, Vol.112(1), 1986, pp. 115-128.

[15] Rakha K.A., Deigaard R., Brøker I., A phaseresolving cross shore sediment transport model for beach profile evolution, Coastal Engineeing, Vol.31(1-4), 1997, pp.231-261.

[16] Roelvink J.A., Reniers A.J.H.M., LIP 11D

Delta Flume experiments, A data set for profile model validation, Report H2130," Delft

Hydraulics, The Netherlands, 1995.

[17] Engelund F., Fredsøe J., A sediment transport model for straight alluvial channels, Nordic Hidrology, Vol. 7, 1976, pp. 293-306.

[18] Gravens M.B., Wang P., Data Report: LSTF Experiments - Transport by Waves and
Currents \& Tombolo Development Behind Headland Structures, Technical Report ERDC/CHLTR-04-9, Coastal and Hydraulics Laboratory, US Army Engineer Research and Development Center Vicksburg MS, 2007. 